科 学 通 报

\title{
岩盐型 $\mathrm{ZnO}$ 纳米微粒的生成和光谱特性
}

\author{
邹炳锁*王斌汤国庆张桂兰陈文驹 \\ (南开大学现代光学研究所, 天津 300071)
}

\section{关键词岩盐型橆化锌、纳米微粒、光谱特征、激子限域、制备}

$\mathrm{ZnO}$ (六角纤锌矿结构) 是一种重要的 II- VI 族化合物半导体, 具有多种用途, 其光学 性质已得到许多结果 ${ }^{[1]} .30$ 年前曾有过它在 $9.5 \mathrm{GPa}$ 下转变成岩盐结构的报道 ${ }^{[2]}$, 最近又有人 ${ }^{[3]}$ 预言 $\mathrm{ZnO}$ 岩盐结构相可能具有独特的光学和输运性质, 并可用于半导体器件, 但直到目前仍 无这方面的结果报道. 本文首次用化学合成方法制备了纳米尺度的岩盐型 $\mathrm{ZnO}$ 微粒, 其光学 性质及结构特征表现了明显类似于激子限域下激子半导体 $\mathrm{Cu}_{2} \mathrm{O}$ 纳米徽粒的性质, 且稳定性 好, 因而有可能成为一种新的光学材料.

\section{1 实验方法}

$\mathrm{ZnO}(\mathrm{R})\{[\mathrm{R}$-rocksalt 岩盐 ],[W-wurtzite 纤锌矿 ] \} 纳米微粒的化学制备流程如下:

$\mathrm{ZnO}(\mathrm{R})$ 或 $(\mathrm{W})$ 纳米微粒有机溶胶.

$$
\mathrm{Zn}^{2+} \text { 水溶液 } \frac{\text { 表面活性剂 }}{\text { 有机溶剂 }} \text { 混合液 } \stackrel{\mathrm{OH}^{-}}{\longrightarrow} \mathrm{Zn}(\mathrm{OH})_{2} \text { 微乳液 } \underset{\text { (回流) }}{\stackrel{\text { 加热 }}{\longrightarrow}}
$$

经此法制得的 $\mathrm{ZnO}(\mathrm{R})$ 微粒经透射电镜 (TEM) 观察为球形纳米尺度, 尺寸为 $4 \mathrm{~nm}$, 我们 用岛津 UV240 型分光光度计测得了它的吸收光谱, 用日立 F4000 荧光分光光度计测得了它的 苂光光谱和激发光谱, 用 Nicolet $170 \mathrm{SX}$ 型红外光谱仪测得了它的红外透射谱, 从中看到了 一些新的现象, 这些现象尚未见报道.

\section{2 结果与讨论}

图 1 是 $\mathrm{ZnO}(\mathrm{W})$ 和 $\mathrm{ZnO}(\mathrm{R})$ 纳米微粒的吸收光谱, 后者较前者的吸收起始位置远远红 移, 可见光区有明显的吸收, 这一显著的变化来自于体系电子结构的变化.

在 $\mathrm{ZnO}(\mathrm{W})$ 中, $\mathrm{Zn}^{2+}$ 以 $4 s p^{3}$ 轨道与 $\mathrm{O} 2 p$ 轨道形成价电子带, $\mathrm{O}$ 的配位数为 $4, \mathrm{Zn}^{2+}$ 的 $3 d$ 轨道几乎不参与价键形成, 此时其光学带隙为 $3.4 \mathrm{eV}$, 而在 $\mathrm{ZnO}(\mathrm{R})$ 中, $\mathrm{Zn}^{2+}$ 以 $3 d-4 s p^{3}$ 杂化 轨道与 $\mathrm{O} 2 p$ 轨道形成价电子带, $\mathrm{O}$ 的配位数为 $6, \mathrm{Zn}^{2+}$ 的 $3 d$ 电子强烈参与成键, 因而其共价 性较前者增强, 键长缩短, 由此从理论上计算得到的直接带隙宽度为 $2.36 \mathrm{eV}^{[3]}$, 远小于 $\mathrm{ZnO}$ (W) 的带隙值 ${ }^{[4]}$, 这与我们的吸收光谱的结果基本上一致.

1993-05-27 收稿, 1993-09-20 收修改稿.

* 现通信地址: 中国科学院物理研究所, 北京 100080 . 

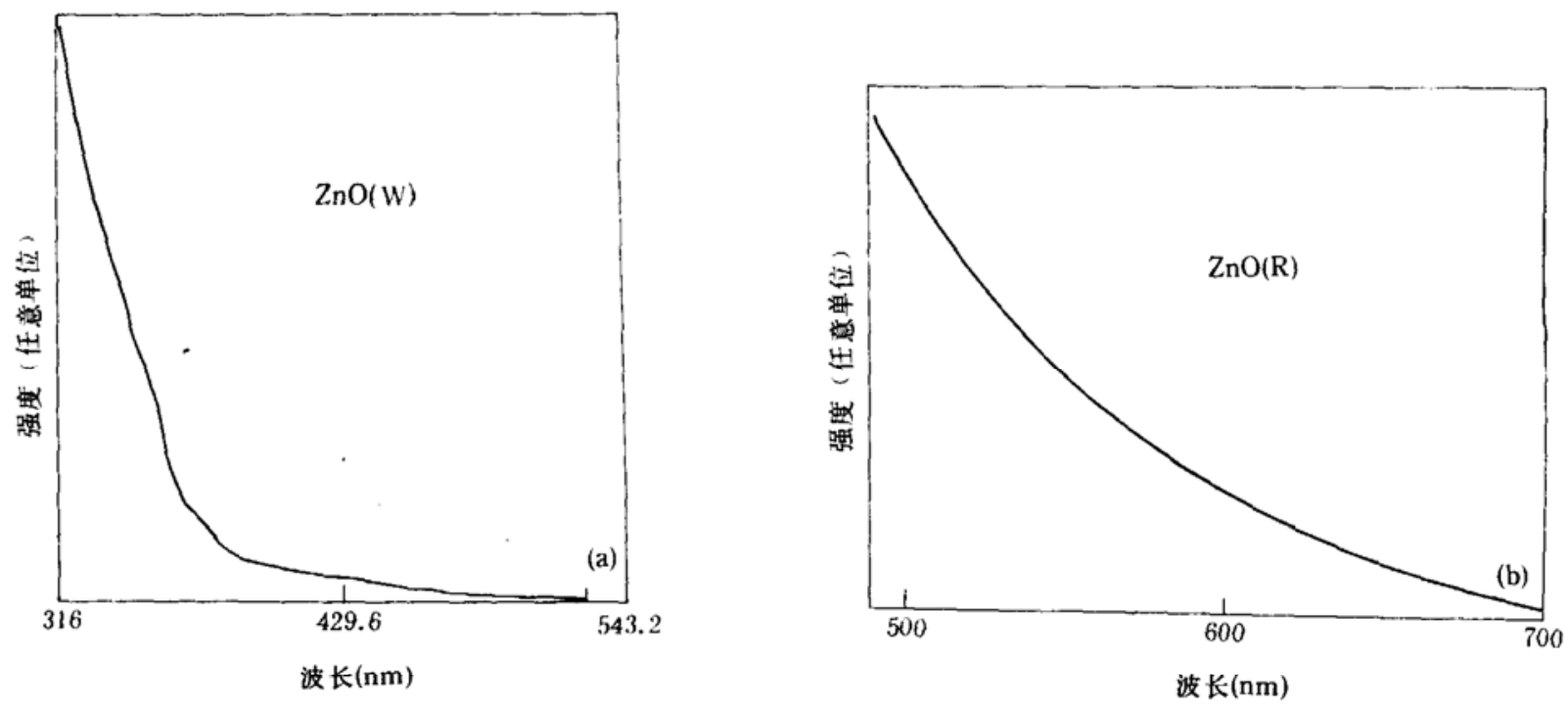

图 $1 \mathrm{ZnO}(\mathrm{W})$ 和 $\mathrm{ZnO}(\mathrm{R})$ 纳米微粒 (约 $4 \mathrm{~nm}$ ) 的吸收光谱

在我们制备的过程中, 最后一步加热 (回流) 如温度在 $250^{\circ} \mathrm{C}$ 以下, 产物为无色透明的 $\mathrm{ZnO}$ (W) 纳米微粒, 升高温度显著超过 $250^{\circ} \mathrm{C}$ 后, 产物变为深红色的 $\mathrm{ZnO}(\mathrm{R})$ 纳米微粒, 冷却到室 温也不变回 $\mathrm{ZnO}(\mathrm{W})$. 事实上 $\mathrm{ZnO}$ 体相表面驰豫态就存在着 $\mathrm{ZnO}(\mathrm{R})$ 相结构 ${ }^{[\Omega]}$, 故而在 $\mathrm{ZnO}(\mathrm{W})$ 纳米微粒中可以发生在表面修饰物 (表面活性剂) 促进的热转变, 从而变成 $\mathrm{ZnO}(\mathrm{R})$ 相, 由此来 看界面极化效应的贡献是不可忽视的 ${ }^{[}$, 界面极化增强将会导致自束缚态 (电子或空穴 - 声子 的束缚态) [self-trapped state] 生成, 当电子-声子的非线性偶合增强到一定程度,就会导致结构 不稳定性 ${ }^{[7}$, 产生相变, 从而造成光学性质的变化.
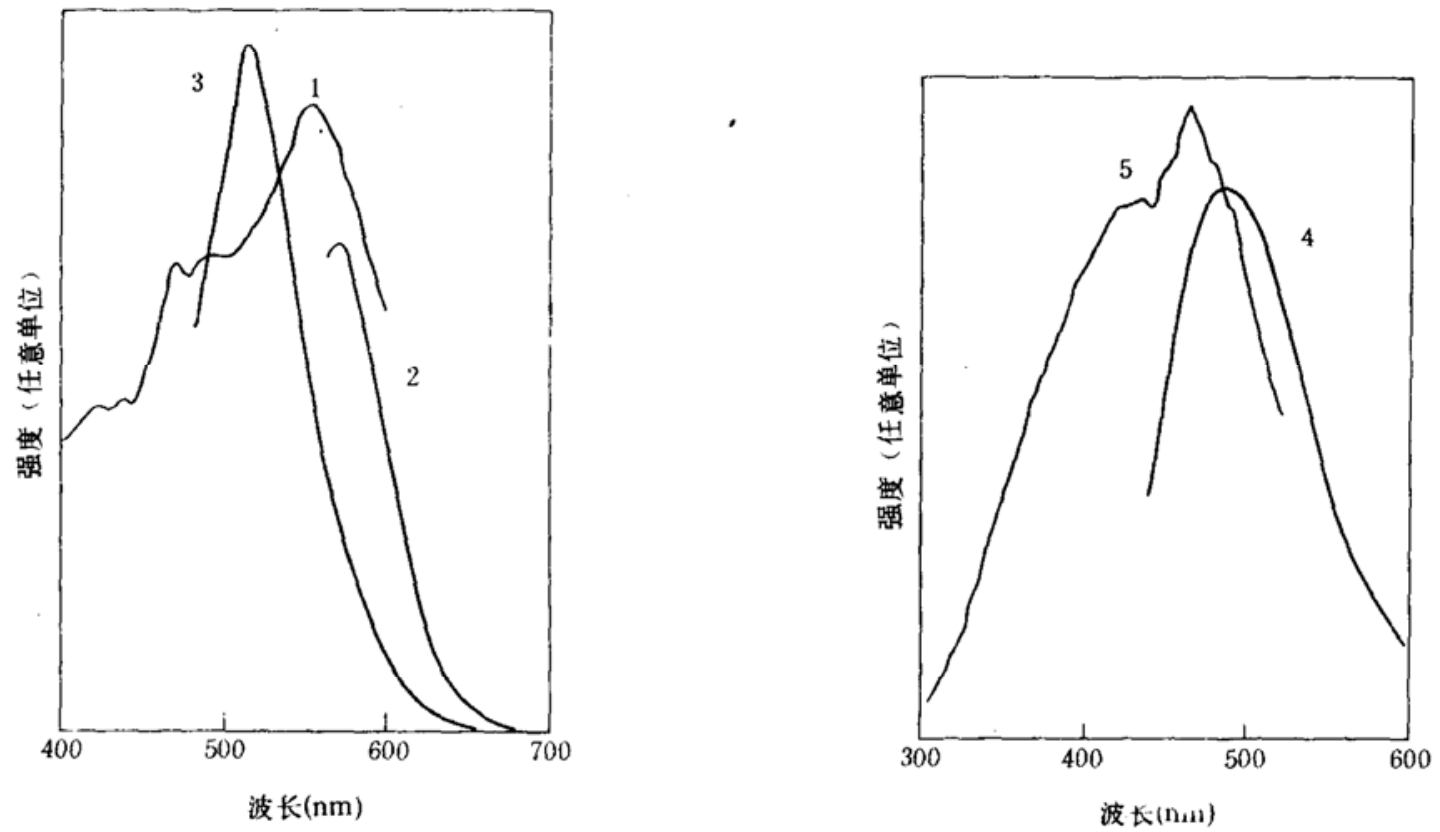

图 $2 \mathrm{ZnO}(\mathrm{R})$ 纳米微粒的菼光光嘴和激发光谱

1 为 $620 \mathrm{~nm}$ 发射时的激发谱, 2 为 $550 \mathrm{~nm}$ 激发时的茨光谱, 3 为 $420 \mathrm{~nm}$ 激发时的苂光谱, 4 为 $420 \mathrm{~nm}$ 激发时的荻光谱, 
图 2 是表面修饰有硬脂酸的 $\mathrm{ZnO}(\mathrm{R})$ 纳米微粒的光致发光谱和激发光谱. 从图中可看到, $\mathrm{ZnO}(\mathrm{R})$ 纳米微粒具有多个共振激子辐射跃迁: (1) $350-445 \mathrm{~nm}$, 紫系列; (2) $450-510 \mathrm{~nm}$, 蓝 系列; (3) $510-580 \mathrm{~nm}$, 黄系列; (4) $580-650 \mathrm{~nm}$, 橙红系列; 激发谱完全类似于 $\mathrm{Cu}_{2} \mathrm{O}$ 纳米微粒 的激子激发结构 ${ }^{[8]}$, 激子跃迁能级分立明显, 这是因为它们具有相同的价键电子态和结构对称 性, 比较接近的电子有效质量, 只不过 $\mathrm{ZnO}$ 具有稍大些的旋轨偶合䢃裂, 即紫系与蓝系的光学 跃迁能量之差稍大些.

Jaffe 等 $^{[3]}$ 利用从头算的相关 Hatree-Fock 模型计算了 $\mathrm{ZnO}(\mathrm{R})$ 的电子结构, 得到了直接带 隙 $(2.36 \mathrm{eV})$ 和间接带隙 $(1.36 \mathrm{eV})$; 图 1 中 $500 \mathrm{~nm}$ 以低能量谱区的吸收很可能就是由于间接跃 迁的贡献. 纳米微粒中的间接带隙跃迁也会发 生光学跃迁 ${ }^{[8,9]}$, 这是由于量子限域效应和介电 限域效应的贡献, 因而 $\mathrm{ZnO}(\mathrm{R})$ 纳米微粒在 $550 \mathrm{~nm}$ 以下能量也能观察到明显的辐射跃迁 (见图 2), 这时界面极化增强的电子 - 声子偶合 也会促进此过程的发生, 从其红外光谱就可看 到此特征.

图 3 是 $\mathrm{ZnO}(\mathrm{R})$ 纳米微粒的红外透射光 谱, 从图中可以看到位于 $300 \mathrm{~cm}^{-1}$ 以下的与 $\mathrm{Zn}^{2+}$ 相关的振动模式成为最强的吸收峰, 最低

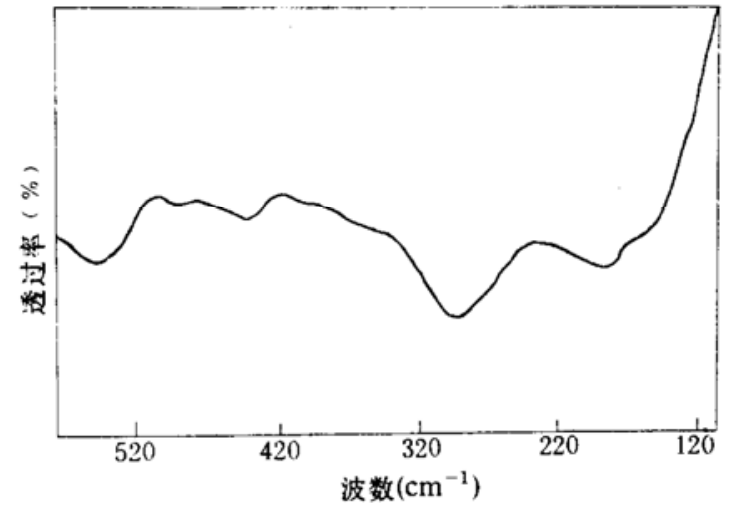

图 $3 \mathrm{ZnO}(\mathrm{R})$ 纳米微粒的红外透射谱 频振动模式以下产生红外带隙, 最高频模式稍微蓝移, 而在 $\mathrm{ZnO}(\mathrm{W})$ 纳米微粒红外光谱中几 乎观察不到 $\mathrm{Zn}^{2+}$ 的振动 (低于 $300 \mathrm{~cm}^{-1}$ ) ${ }^{[10]}$, 这直接证明了: (1) 体系中电子 - 声子确实存在强 烈的相互作用, (2) 价电子带中 $\mathrm{Zn}^{2+} 3 d$ 电子的成键作用, 这样才能解释与 $\mathrm{Zn}^{2+}$ 相关的振动模 式的显著增强, 当然这些变化还需要进一步定量的理论描述.

总之, 本文首次报道了 $\mathrm{ZnO}(\mathrm{R})$ 纳米微粒的光谱特性和电子结构特征, 实验表明它是一种 具有激子半导体性质的新材料, 稳定性好, 肯定会在非线性光学材料性质和应用方面起重要 的作用.

\section{参 考文 献}

[1] Butkhuzi, T. V., Geogobiani, A. N., Ye, Z. U. et al., Luminescence of Wide-band Semiconductors (ed. Galanin, M. D.), Nova Scienœe Publishers, New York, 1990, 167-216.

[ 2] Bates, C. H., White. W. B., Roy, R., Science, 1962, 137: 993-996.

[ 3] Jaffe, J. E., Pandey, R., Kunx, A. B., Phys. Rev., B, 1991, 43: 14030-14036.

[4] Liang, W. Y., Yoffe, A. D., Phys. Rev. Lett., 1968, 20:5-9.

[ 5] Skinner, A. J., Lafemina, J. P., Phys. Rev., B, 1992, 45: 4557-4562.

[ 6] Banyai, L., Cilliot, J. P. et al., Phys. Rev., B, 1992, 45: 14136-14144.

[ 7] de Bodas, E. L., Hipplito, O., Phys. Rev., B, 1983, 27:6110-6123.

[8] 邹炳锁等,科学通报, 1993, 38(12): 1183-1185.

[ 9] Takagahara, T., Takeda, K., Phys. Rev., B, 1992, 46: 15578-15583.

[10] Hayashi, S., Nakamcri, N., Kanamori, H., J. Phys. Soc. Jpn. 1979, 46: 176-183. 